\title{
First Principle Study on Atomic Scale Structures of Cathode in Aluminium-ion Battery Using Various van der Waals Corrections
}

\author{
Kaihao Geng ${ }^{1}$, Haining $\mathrm{Cao}^{1 *}$ and Meng-Chang $\operatorname{Lin}^{1}$ \\ ${ }^{1}$ Key Laboratory for Robot and Intelligent Technology of Shandong Province, College of Electrical Engineering and Automation, \\ Shandong University of Science and Technology, Qingdao, Shandong, 266590, China
}

\begin{abstract}
There is still controversy on the atomistic configuration of aluminium-ion batteries (AIB) cathode when using first principle calculation based on density functional theory (DFT). We examined the relevant cathodic structures of $\mathrm{Al} /$ graphite battery by employing several van der Waals (vdW) corrections. Among them, DFT-TS method was determined to be a better dispersion correction in correctly rendering structural features already found through experiment investigations. The systematic comparison paved the way to the choice of vdW parameters in first principle calculation of graphitic electrode.
\end{abstract}

\section{Introduction}

In recent years, with the increase in energy demand and the rapid development of the electronics industry, there is an urgent need for clean and efficient energy storage equipment to meet the needs of intermittent production and wearable equipment. Currently widely used lithiumion batteries face bottlenecks due to the scarcity of lithium in the earth's crust and the inherent shortcomings of single-electron discharge characteristics[1], and, meanwhile, other metal anode materials, such as sodium, magnesium, potassium, calcium, and aluminium, are considered potential candidates for electrochemical energy storage devices. Among them, aluminium has unique advantages in cost and efficiency due to its high abundance in the earth's crust and three-electron redox characteristics[2][3]. Based on three electron transfer electrode reaction $\left(\mathrm{Al} \leftrightarrow \mathrm{Al}^{3+}+3 \mathrm{e}^{-}\right)$, aluminium metal can provide up to $2980 \mathrm{mAh} / \mathrm{g}$ gravimetric capacity and $8040 \mathrm{mAh} / \mathrm{cm}^{3}$ volumetric capacity[4]. Since Lin[5] et al. made the breakthrough of Al-ion battery, there have been many controversies about the reaction mechanism on the cathode side. This Al-ion battery used aluminium foil as the anode, three-dimensional graphite foam as the cathode, and [EMIm]Cl mixed with $\mathrm{AlCl}_{3}$ as the ionic liquid electrolyte[6]. Due to the structural complexity of graphite, how $\mathrm{AlCl}_{4}$ clusters interplay with the cathode of the $\mathrm{Al} /$ graphite battery seems to be a perplexing problem.

In order to explore the mechanism of $\mathrm{AlCl}_{4}$ intercalation in graphite cathode of $\mathrm{Al} /$ graphite batteries and explain the evolution of the graphite interlayer compounds (GICs)[7] structure after $\mathrm{AlCl}_{4}$ is intercalated in graphite, $\mathrm{Wu}[8]$ et al. first proposed a planar quadrangle configuration of $\mathrm{AlCl}_{4}$. They adjusted the van der Waals interaction in the system by adjusting the $\mathrm{C}_{6}$ parameter in Grimme's DFT-D2 method, so that the optimized original graphite layer spacing $(3.353 \AA)$ was close to the experimental value (3.336 $)$. They believe that all van der Waals correction related methods are based on experience, so the standard parameters in Grimme's DFT-D2 method can be changed according to the actual system. However, in the calculation using adjusted DFT-D2 method, the configuration of $\mathrm{AlCl}_{4}$ in the graphite interlayer compound after optimization is a planar quadrangle geometry.

Jung[9], Bhauriyal[10], Gao[11], Wang[12], Li[13] and other researchers believe that the $\mathrm{AlCl}_{4}$ inserted into the graphite layer should be a tetrahedral configuration. Jung and others put forward different opinions on the work of Wu and others, and they tested DFT-D2, DFTD3(zero-damping), DFT-D3(BJ-damping), vdWDF(revPBE), vdW-DF(optPBE), vdW-DF(optB88), vdW-DF(optB86b), vdW-DF(PW86R) these eight dispersion correction methods are used to describe van der Waals force. During the test, the cut-off energy is set at $400 \mathrm{eV}$. Among them, the DFT-D2 method is poor in describing the graphite layer spacing and the calculated pristine graphite layer spacing is $3.2 \AA$. And the remaining methods obtain the original graphite layer spacing sufficiently accurate, about 3.25 3.35 . Especially DFT-D3(zero-damping), vdW-DF(revPBE), vdW-DF(optPBE), vdW-DF2(PW86R) methods, the difference from the experimental value of the layer spacing is less than $0.03 \AA$, and the optimized $\mathrm{AlCl}_{4}$ tends to tetrahedron configuration, $0.36 \sim 0.77 \mathrm{eV}$ more stable than planar quadrangle configuration $\mathrm{AlCl}_{4}$.

*Corresponding author's e-mail: caohn@sdust.edu.cn 


\section{Calculation details}

This work uses a first-principles calculation method based on density functional theory (DFT), calculation using the Vienna ab initio simulation package (VASP) software package. Using projector augmented-wave method (PAW) to describe the interaction between valence electrons and ion reals. In order to describe the exchange-correlation potential, the generalized gradient approximation of Perdew-Burke-Ernzerhof (GGA-PBE) were used. Plane waves energy cut-off optimization result is $600 \mathrm{eV}$. The Brillouin zone integral uses a Gamma centered $4 \times 4 \times 4$ k-point grid. In the process of structural relaxation, the lattice volume and atomic position are fully relaxed until the Hellmann-Feynman force on all atoms is less than $1 \times 10^{-5} \mathrm{eV} / \AA$, total energy error is less than $1 \times 10^{-6} \mathrm{eV}$. To describe the van der
Waals (VDW) interactions, the Tkatchenko-Scheffler (DFT-TS) method was used.

\section{Results and discussion}

\subsection{Calculation model}

We first solved the problem of selecting the van der Waals parameters for calculating the graphite system. The graphite layers are stacked together by van der Waals force. In order to obtain the accurate pristine graphite interlayer spacing, four van der Waals force correction methods, such as DFT-D2[14], DFT-D3(zerodamping)[15], DFT-D3(BJ-damping)[16], DFT-TS[17] were tested, respectively, the data are shown in table 1 .

Table 1. Approximate vdW correction methods with optimized graphite interlayer spacings.

\begin{tabular}{lcc}
\hline Method & Plane wave cut-off energy $(\mathrm{eV})$ & $\mathrm{d}(\AA)$ \\
DFT-D2 & 600 & 3.25 \\
DFT-D3(zero-damping) & 500 & 3.453 \\
DFT-D3(BJ-damping) & 600 & 3.311 \\
DFT-TS & 600 & 3.335 \\
\hline
\end{tabular}

The graphite interlayer spacing optimized by the DFT-D2 method converges when the cut-off energy set at $600 \mathrm{eV}$, which is $3.250 \AA$, it's $0.086 \AA$ lower compared with experimental value $(3.336 \AA)$. The graphite interlayer spacing optimized by the DFT-D3(zerodamping) method converges when the cut-off energy is set at $500 \mathrm{eV}$, which is $3.453 \AA$, it's $0.117 \AA$ higher than the experimental value. When we test the DFT-D3(BJdamping) method, we found that as the plane wave cutoff energy increases, the graphite interlayer spacing fluctuates in the range of 3.306 3.348 $\AA$. Although the graphite interlayer spacing can be optimized to $3.311 \AA$ at $600 \mathrm{eV}$, this fluctuation is very detrimental to the accuracy of the calculation results. Therefore, we tested the DFT-TS method. When the cut-off energy is set at
$450 \mathrm{eV}$ or higher, the graphite interlayer spacing converges near $3.34 \AA$. The difference from the experimental value is about $\sim 0.001 \AA$. So we believe that the DFT-TS method is the best way to describe the van der Waals force between graphene layers. The graphite interlayer spacing after relaxation is $3.335 \AA$, which is approximately equal to the experimental value of $3.336 \AA$ at $4.2 \mathrm{~K}[18]$.

As shown in figure 1 , the optimized graphite configuration is periodically extended to a $3 \times 3 \times 1$ supercell (with $36 \mathrm{C}$ atoms), which simulates the $\mathrm{AB}$ stacked graphite of stage-2, and the stage-n of GIC refers to every other layer of graphene separated by an insert $\mathrm{AlCl}_{4}$ cluster. The follow-up work was carried out in the graphite interlayer compound of stage- 2 .

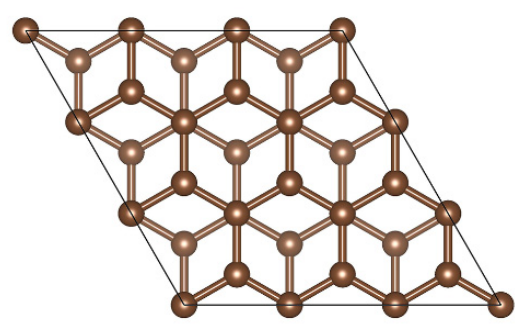

Figure 1. $3 \times 3 \times 1 \mathrm{AB}$ stacks graphite supercell configuration.

\subsection{The geometry of $\mathrm{AlCl}_{4}$ inserted into $\mathrm{GIC}$}

First, to understand which tetrahedral $\mathrm{AlCl}_{4}$ or planar $\mathrm{AlCl}_{4}$ is more stable in the electrolyte of $\mathrm{Al} /$ graphite battery. We calculated the structure of gas phase $\mathrm{AlCl}_{4}$ with tetrahedral and planar geometry in the vacuum, respectively, as shown in figure 2. The tetrahedron geometry of $\mathrm{AlCl}_{4}$ is $0.82 \mathrm{eV}$ more stable than planar geometry of $\mathrm{AlCl}_{4}$. The height of the optimized tetrahedral configuration $\mathrm{AlCl}_{4}$ is about $2.5 \AA$, and the $\mathrm{Al}$ $\mathrm{Cl}$ bond length is $2.15 \AA$. The bond length of $\mathrm{Al}-\mathrm{Cl}$ in planar configuration $\mathrm{AlCl}_{4}$ is $2.18 \AA$. 
(a)

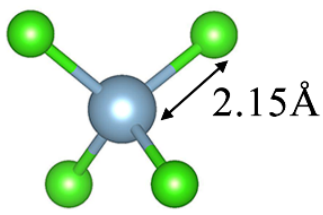

(b)

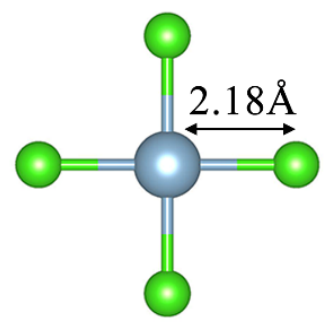

Figure 2. gas phase $\mathrm{AlCl}_{4}$ with (a) tetrahedral and (b) planar geometry in the vacuum.

Previous studies have shown that the distance between the $\mathrm{Cl}$ atom in insert $\mathrm{AlCl}_{4}$ and the $\mathrm{C}$ atom in graphite should be greater than $3 \AA$. In order to accommodate $\mathrm{AlCl}_{4}$ clusters between graphene layers, the graphite interlayer spacing will inevitably expand. In order to investigate whether $\mathrm{AlCl}_{4}$ tends to tetrahedral configuration or planar configuration between graphene layers, we put a tetrahedral configuration of $\mathrm{AlCl}_{4}$ between graphene layers. We used DFT-D3(zerodamping) and DFT-TS van der Waals force correction method to optimize this structure. There are obvious differences in the configuration after relaxation, the $\mathrm{AlCl}_{4}$ between the graphene layers obviously changes to a planar configuration by using DFT-D3(zero-damping) method, and the graphene interlayer spacing becomes $6.41 \AA$, as shown in figure 3(a). Then by using DFT-TS method, the $\mathrm{AlCl}_{4}$ between the graphene layers still
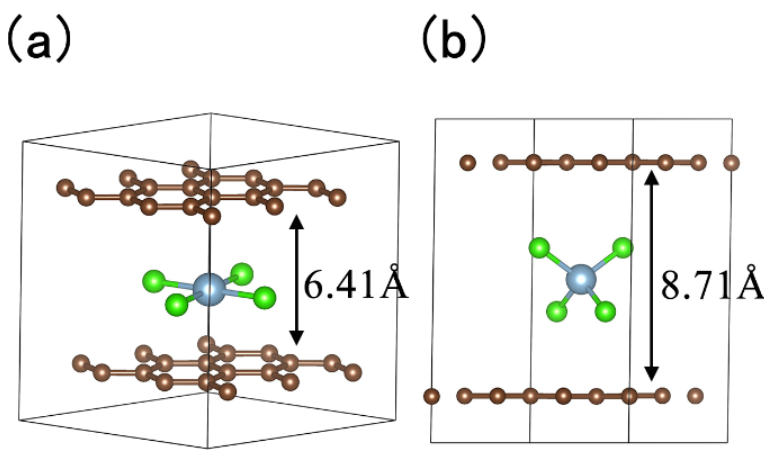

(c)

maintains the tetrahedral configuration, and the graphite interlayer spacing increases to $8.71 \AA$, as shown in figure 3(b)(c). In previous research, Wang[19] et al. observed the tetrahedral coordinated $\mathrm{Al}$ between graphene layers through X-ray absorption spectra, which is in good agreement with the tetrahedral configuration $\mathrm{AlCl}_{4}$ we calculated. This agreement serves as weighty evidence that the DFT-TS method is more accurate in describing the structure of this GICs. Based on this, we mainly studied two tetrahedral configurations of $\mathrm{AlCl}_{4}$ in graphite intercalation: $\mathrm{AlCl}_{4}$ with a 2-fold rotation symmetry about the direction normal to the graphite basal plane(2FR-shape), as shown in figure 3(b); $\mathrm{AlCl}_{4}$ with a 3-fold rotation symmetry about the direction normal to the graphite basal plane(3FR-shape), as shown in figure $3(\mathrm{c})$.

Figure 3. (a) Optimized GIC structure by DFT-D3(zero-damping) method. DFT-TS method optimized GICs structure; (b) $\mathrm{AlCl}_{4}$ with a 2-fold rotation symmetry about the direction normal to the graphite basal plane; (c) $\mathrm{AlCl}_{4}$ with a 3-fold rotation symmetry about the direction normal to the graphite basal plane.

In terms of energy, the GIC with planar geometry $\mathrm{AlCl}_{4}$ between the graphite layers is about $2 \mathrm{eV}$ higher than GICs with tetrahedral geometry $\mathrm{AlCl}_{4}$ between the graphite layers. For tetrahedral geometry $\mathrm{AlCl}_{4}$, the GIC

Table 2. Structural parameters of optimized GICs, interlayer distance between graphene layers $(\mathrm{d})$, bond lengths $\left(\mathrm{d}^{\mathrm{Al}-\mathrm{Cl}}\right)$, bond angle $\left(\theta^{\mathrm{Cl}-\mathrm{Al}-\mathrm{Cl}}\right)$, and the height of $\mathrm{AlCl}_{4}\left(\mathrm{~d}^{\mathrm{AlCl} 4}\right)$.

\begin{tabular}{lccccc}
\hline $\begin{array}{l}\text { Geometry of } \mathrm{AlCl}_{4} \\
\text { in GIC } \\
\text { planar }\end{array}$ & $\begin{array}{c}\text { Lattice parameters }(\AA) \\
\text { aFR }\end{array}$ & $\mathrm{d}(\AA)$ & $\mathrm{d}^{\mathrm{Al}-\mathrm{Cl}}(\AA)$ & $\theta^{\mathrm{Cl}-\mathrm{Al}-\mathrm{Cl}\left({ }^{\circ}\right)}$ & $\mathrm{d}^{\mathrm{AlCl}}(\AA)$ \\
$\mathrm{C}=9.735$ & & & & \\
3FR & $\mathrm{a}=\mathrm{b}=7.375 ; \mathrm{c}=12.06$ & 8.714 & 2.155 to 2.158 & 107.5 to 114.32 & 2.366 \\
\hline
\end{tabular}

with $2 \mathrm{FR}$ shape $\mathrm{AlCl}_{4}$ is about $0.1 \mathrm{eV}$ more stable than the $\mathrm{GIC}$ with 3FR shape $\mathrm{AlCl}_{4}$, the structural parameters are shown in table 2. 


\section{Conclusion}

Investigation of cathode configuration in $\mathrm{Al} /$ graphite batteries is of great significance for development of more capable energy storage system. The DFT-TS method we used to correct the van der Waals force can well describe the graphite interlayer spacing and the GICs structure. Furthermore, we found that the most stable configuration of $\mathrm{AlCl}_{4}$ between graphite layers is $2 \mathrm{FR}$ shape.

\section{References}

1. Wu, F., Yang, H.Y., Bai, Y., et al. (2019) Paving the Path toward Reliable Cathode Materials for Aluminium-Ion Batteries. Advanced Materials, 31: 1806510 .

2. Shyamal, K.D., Sadhan, M., Homen L. (2017) Aluminium-ion batteries: developments and challenges. J. Mater. Chem. A, 5: 6347-6367.

3. Lv, Z.C., Han, M., Sun, J.H., et al. (2019) A high discharge voltage dual-ion rechargeable battery using pure $\left(\mathrm{DMPI}^{+}\right)\left(\mathrm{AlCl}_{4}^{-}\right)$ionic liquid electrolyte. Journal of Power Sources, 418: 233240 .

4. Giuseppe, A.E., Krystan, M., Katrin, H., et al. (2016) An Overview and Future Perspectives of Aluminium Batteries. Advanced Materials, 28: 7564-7579.

5. Lin, M.C., Gong, M., Lu, B.A., et al. (2015) An ultrafast rechargeable aluminium-ion battery. Nature, 520: 324-328.

6. Pan, C.J., Yuan, C.Z., Zhu, G.Z., et al. (2018) An operando X-ray diffraction study of chloroaluminate anion-graphite intercalation in aluminium batteries. Proceedings of the National Academy of Sciences, 115: 5670-5675.

7. Haegyeom, K., Jihyun, H., Gabin, Y., et al. (2015) Sodium intercalation chemistry in graphite. Energy \& Environmental Science, 8: 2963-2969.

8. Wu, M., Xu, B., et al. (2016) Geometry and fast diffusion of $\mathrm{AlCl} 4$ cluster intercalated in graphite. Electrochimica Acta, 195: 158-165.

9. Jung, S.C., Kang, Y.J., Yoo, D.J., et al. (2016) Flexible Few-Layered Graphene for the Ultrafast Rechargeable Aluminium-Ion Battery. J. Phys. Chem. C, 120: 13384-13389.

10. Preeti, B., Arup, M., Biswarup, P. (2017) The staging mechanism of $\mathrm{AlCl} 4$ intercalation in a graphite electrode for an aluminium-ion battery. Phys. Chem. Chem. Phys., 19: 7980-7989.

11. Gao, Y.R., Zhu, C.Q., Chen, Z.Z., et al. (2017) Understanding Ultrafast Rechargeable Aluminium-Ion Battery from First-Principles. J. Phys. Chem. C, 121: 7131-7138.

12. Wang, Q.P., Zheng, D.Y., He, L.X., et al. (2019) Cooperative Effect in a Graphite Intercalation
Compound: Enhanced Mobility of $\mathrm{AlCl} 4$ in the Graphite Cathode of Aluminium-Ion Batteries. Phys. Rev. Applied, 12: 044060.

13. Li, J., Liu, Q., Lemmon, J.P., Flores, R.A., Bligaard, T. (2020) DFT simulation of the X-ray diffraction pattern of aluminium-ion-intercalated graphite used as the cathode material of the aluminium-ion battery. Phys. Chem. Chem. Phys., 22: 5969-5975.

14. Grimme, S. (2006) Semiempirical GGA-type density functional constructed with a long-range dispersion correction. J. Comput. Chem, 27: 1787-1798.

15. Grimme, S., Antony, J., Ehrlich, S., Krieg, H. (2010) A consistent and accurate ab initio parametrization of density functional dispersion correction (DFT-D) for the 94 elements H-Pu. J. Chem. Phys., 132: 154104.

16. Grimme, S., Ehrlich, S., Goerigk, L. (2011) Effect of the damping function in dispersion corrected density functional theory. J. Comput. Chem., 32: 1456-1465.

17. Tkatchenko, A., Scheffler, M. (2009) Accurate molecular van der waals interactions from ground-state electron density and free-atom reference data. Phys. Rev. Lett., 102: 073005.

18. Baskin, Y., Meyer, L. (1955) Lattice Constants of Graphite at Low Temperatures. Physical Review., 100: 544.

19. Wang, D.Y., Huang, S.K., Liao, H.J., et al. (2019) Insights into dynamic molecular intercalation mechanism for AlC battery by operando synchrotron X-ray techniques. Carbon, 146: 528534. 